\title{
Microphase Separation of Poly(tert-Butyl Methacrylate)-block-Polystyrene Diblock Copolymers to Form Perforated Lamellae
}

\author{
Dongdong Yao, ${ }^{\mathrm{b}}$ Ke Zhang, ${ }^{\mathrm{b}}$ and Yongming Chen*ab \\ ${ }^{a}$ Key Laboratory for Polymeric Composite and Functional Materials of Ministry of \\ Education, Guangdong Provincial Key Laboratory for High Performance \\ Polymer-based Composites, Department of Polymer and Material Sciences, School of \\ Chemistry and Chemical Engineering, Sun Yat-Sen University, Guangzhou 510275, \\ China. \\ ${ }^{b}$ Laboratory of Polymer Physics and Chemistry, Institute of Chemistry, The Chinese \\ Academy of Sciences, Beijing 100190, China. \\ * Corresponding Author. Y. M. Chen, E-mail: chenym35@mail.sysu.edu.cn
}

\begin{abstract}
Perforated lamellar (PL) microphase structure of block copolymers in the bulk is rarely observed. Herein, formation of the PL structure from simple block copolymer, poly(tert-butyl methacrylate)-block-polystyrene (PtBMA- $b$-PS) prepared by reversible addition-fragmentation chain transfer (RAFT) mediated radical polymerization, is reported. The PL phase consists of alternating layers of PS and P $t$ BMA microdomains, among which the PS layers contain ordered cylindrical domains of PtBMA. It is confirmed that the PL phase is located between the cylindrical phase and lamellar phase. In methanol, the microphase-separated sample is readily split into individual core/shell nanosheets with a perforated glassy PS core. The PtBMA domains are densely attached both as the shells along PS sheets and as passages with a hexagonal pattern. The passage has a diameter of ca. $12 \mathrm{~nm}$ and an average periodic distance of $31 \mathrm{~nm}$.
\end{abstract}

Keywords Block copolymer, Bulk microphase separation, Perforated lamellar structure, Perforated nanosheets. 


\section{Introduction}

Block copolymers consisting of incompatible polymer segments linked by covalent bonding may undergo microphase separation in the bulk to form a variety of novel materials with ordered periodic nanostructures [1-3]. This property endows microphase-separated block copolymer materials with a great potential application for developing advanced materials and technology in application like lithography, catalysis and filtration [4-7]. It is well-known that bulk microphase separation behaviors of block copolymers are affected mainly by polymerization degree $(N)$, composition $(f)$, and Flory-Huggins interaction parameter $(\chi)$, etc. $[1,2,8]$ In general, AB diblock copolymers, the simplest architecture of block copolymers, form four typical equilibrium phase structures: lamella (L), cylinder (C), gyroid and sphere, dependent upon the polymer composition or volume fraction of two segments [2]. With the increase of complexity of block copolymer architecture, much more nanostructures may be obtained [8-10]. However, it is more attractive to obtain complex structures from simple diblock copolymers. The metastable morphologies have been often observed in literatures but obtained much less attention. Among them, the perforated lamella (PL) has a very interesting structure that was reported rarely [11-22]. Such atypical PL phase structure consists of alternating layers of $\mathrm{A}\left(f_{\mathrm{A}}<0.5\right)$ and $\mathrm{B}\left(f_{\mathrm{B}}=1-f_{\mathrm{A}}\right)$ components in asymmetric $\mathrm{AB}$ block copolymers and the A layers contain an ordered cylindrical domain of B component [13, 16, 22]. It is believed to be a transition phase between $\mathrm{C}$ and $\mathrm{L}$ phase, and it occupies a very narrow region near order-disorder transition in the phase diagram of block copolymer bulks [23, 24].

In the early period, Bates et al. captured the hexagonal PL phase from the microphase-separation of polyisoprene-block-polystyrene (PI-b-PS) diblock copolymers $\left(f_{\mathrm{PI}}=0.38, M_{\text {total }}=31.5 \mathrm{~kg} \mathrm{~mol}^{-1}\right)$, in which one layer is formed by PS, while the other layer consists of perforated PI layer filled with PS cylindrical domains [23, 24]. Later, Loos and coworkers found the PL structure in polystyrene-block-poly(4-vinylpyridine)/pentadecylphenol (PS- $b-\mathrm{P} 4 \mathrm{VP} / \mathrm{PDP})$ supramolecular system only when the P4VP/PDP block fraction is at $0.31 \sim 0.37$ [16]. 
Very recently, PL phase was observed from bulk materials of polybutadiene-block-poly(2-vinylpyridine)-block-poly(tert-butyl methacrylate) triblock terpolymers by Müller, et al. $[11,12]$. The PL phase of block copolymers is an interesting structure in which one perforated layer is isolated by another layer that is connected through pores. This structure may be important for developing novel materials with unique porous nanostructure. However, as far as we knew, the observation and study of PL structure are reported rarely because of its kinetic controlled structure. Additionally, almost all of the block copolymers that form PL structures are given by anionic polymerization [11-20].

Herein we report the PL phase formation from a kind of very simple diblock copolymers that are synthesized by reversible addition-fragmentation transfer (RAFT) radical polymerization, which provides a great convenience for post-modification and functionalization of materials. We found that the bulk microphase separation of poly(tert-butyl methacrylate)-block-polystyrene (PtBMA-b-PS) diblock copolymers formed PL phase between the L and C phase by tuning composition of the copolymers. As shown in Scheme 1, the alternating layers of PS and PtBMA are formed and the PS layers contain the ordered domains of P $t$ BMA. Moreover, the individual core/shell perforated nanosheets are obtained by direct dispersing the PL sample into methanol at room temperature. Furthermore, the water-dispersed perforated nanosheets are obtained by hydrolyzing PtBMA into poly(methacrylic acid) (PMAA) using trifluoroacetic acid (TFA), showing unique sheetlike nanoobjects with a patterned internal structure in water. 


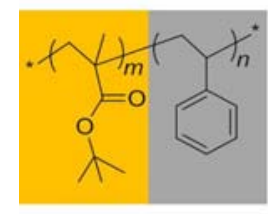

PtBMA- $b$-PS

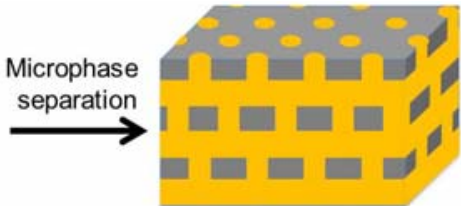

Perforated Lamellae

Dispersing

\section{PS}

PtBMA

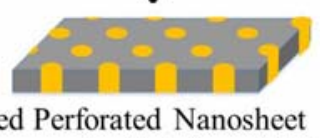

Isolated Perforated Nanosheet

(Both sides covered with tethered PtBMA)

Scheme 1. Schematic illustration of the formation of perforated lamellar microphase structure and perforated nanosheets from PtBMA- $b$-PS diblock copolymers. Yellow and deep gray colors represent PtBMA and PS components, respectively.

\section{Experimental section}

\subsection{Materials}

Styrene (St, $>99 \%$, Aldrich) and tert-butyl methacrylate ( $t$ BMA, 98\%, Aldrich) were

distilled from calcium hydride under reduced pressure, respectively. Azobis isobutyronitrile (AIBN, 99\%, Aldrich) was recrystallized from ethanol and dried. Cumyl dithiobenzoate (CDB) was synthesized according to the previous literature [25]. Tetrahydrofuran (THF) as reaction solvent for polymerization were refluxed several hours and freshly distilled from sodium benzophenone ketyl solution prior to use. All other chemicals such as trifluoroacetic acid (TFA) and methanol were analytical grade and obtained from commercial sources.

\subsection{Characterization}

${ }^{1} \mathrm{H}$ NMR spectra were recorded on a Bruker DMX400 spectrometer with $\mathrm{CDCl}_{3}$ as solvent at $25{ }^{\circ} \mathrm{C}$. Molecular weight distribution $\left(M_{\mathrm{w}} / M_{\mathrm{n}}\right)$ of the polymers were determined by size exclusion chromatography (SEC), equipped with a Waters 515 HPLC pump and a Waters 2414 refractive index detector, with DMF containing $1.0 \mathrm{~g}$ $\mathrm{L}^{-1}$ of $\mathrm{LiBr}$ as the eluent and a $1.0 \mathrm{~mL} \mathrm{~min}^{-1}$ flow rate at $50{ }^{\circ} \mathrm{C}$. The results were calibrated with polystyrene standards. Small-angle X-ray scattering (SAXS) data was 
collected on a Rigaku Micro Max-007 HF copper rotating-anode operated at $40 \mathrm{kV}$ and $30 \mathrm{~mA}$. Wavelength of the incident $\mathrm{X}$-ray beam from $\mathrm{Cu} \mathrm{K \alpha}$ radiation was 0.154 nm. Transmission electron microscopy (TEM) images were obtained on a Hitachi $\mathrm{H}-800$ instrument operated at an accelerating voltage of $100 \mathrm{kV}$, and the images were recorded by a digital camera. Scanning electron microscopy (SEM) images were obtained on JSM-6700F instrument with an acceleration voltage of $5 \mathrm{kV}$. FT-IR spectra were recorded on Thermo Nicolet Avatar-330 spectrometer, and samples were prepared as a thin pellet with potassium bromide. Atomic force microscopy (AFM) image was recorded using a Multimode 8 (Bruker) AFM in tapping mode under ambient conditions. Samples were prepared by spin coating methanol dispersion $(0.5$ $\mathrm{mg} \mathrm{mL} \mathrm{m}^{-1}$ ) on mica with a rate of $2000 \mathrm{rpm}$.

\subsection{Synthesis of PtBMA-b-PS diblock copolymers}

PtBMA- $b$-PS diblock copolymers with different polymeric degree of PS were prepared using a procedure of two-step reversible addition-fragmentation transfer (RAFT) mediated radical polymerization. In the first step, PtBMA macro-chain transfer agent (macroCTA) was synthesized by CDB-mediated RAFT polymerization. AIBN (7.7 mg, $0.047 \mathrm{mmol}), \mathrm{CDB}$ (63.7 mg, $0.234 \mathrm{mmol}), t$ BMA (10.0 g, $70.3 \mathrm{mmol})$ and THF ( $4.30 \mathrm{~g})$ were added into a dry $20 \mathrm{~mL}$ glass ampule containing a stirring bar. The mixture was degassed by three freeze-pump-thaw cycles. Subsequently, the ampule was sealed under vacuum. The polymerization reaction was carried out at 60 ${ }^{\circ} \mathrm{C}$ for $14 \mathrm{~h}$. After cooling to room temperature, the crude product was diluted by THF and purified by precipitating into an excess amount of methanol/water $(\mathrm{v} / \mathrm{v}=2 / 1)$ three times and then dried in a vacuum oven overnight at $40{ }^{\circ} \mathrm{C}$ to give pink powder. The $t$ BMA monomer conversion (75.6\%) was calculated according to ${ }^{1} \mathrm{H}$ NMR spectrum of crude product in $\mathrm{CDCl}_{3}$, and molecular weight distribution $\left(M_{\mathrm{w}} / M_{\mathrm{n}}\right)$ was

determined by SEC with DMF as eluent. Product: $\mathrm{P} t \mathrm{BMA}_{227}, M_{\mathrm{n}, \mathrm{NMR}}=32500, M_{\mathrm{w}} / M_{\mathrm{n}}$ $=1.07$.

Then, a series of $\mathrm{P} t \mathrm{BMA}_{227}-b-\mathrm{PS}_{\mathrm{n}}$ diblock copolymers with different PS chain length were synthesized using $\mathrm{P} \mathrm{BMA}_{227}$ as a macroCTA. The typical procedure was 
as follows. The PtBMA 227 macroCTA $(2.01 \mathrm{~g}, 0.062 \mathrm{mmol})$ and $\mathrm{St}(4.47 \mathrm{~g}, 43.0 \mathrm{mmol})$ were added into a $10 \mathrm{~mL}$ glass ampule. A similar experimental setup was then performed as that described above. The reaction was carried out at $90{ }^{\circ} \mathrm{C}$ for $27 \mathrm{~h}$. The crude product was dissolved in THF and precipitated into methanol three times. After drying, the pink powder was obtained. The samples were characterized by SEC and ${ }^{1} \mathrm{H}$ NMR, respectively.

\subsection{Bulk microphase-separation of PtBMA-b-PS diblock copolymers}

The microphase-separated samples were prepared by solution casting method. The P $t$ BMA- $b$-PS diblock copolymers with different composition were dissolved in THF with a concentration of $50.0 \mathrm{mg} \mathrm{mL}^{-1}$ and then poured into a clean Teflon substrate, respectively. The solvent was allowed to slowly evaporate in a vacuum desiccator for about four days. The film samples were then dried overnight in a vacuum oven at 30 ${ }^{\circ} \mathrm{C}$. At last, the samples were thermally annealed under nitrogen atmosphere in an oil bath at $155^{\circ} \mathrm{C}$ for five days.

\subsection{Preparation of isolated polymeric nanoobjects}

The bulk microphase-separated samples were immersed into methanol with a concentration of $1.0 \mathrm{mg} \mathrm{mL}^{-1}$ and stirred vigorously for approximately one day. The bulk samples disappeared and homogeneous dispersions were obtained, and the morphologies of dispersions were mainly observed by TEM.

\subsection{Hydrolysis of PtBMA component of perforated nanosheets}

Trifluoroacetic acid (TFA) (50-fold excess to $t$ BMA) was added into methanol dispersions of perforated nanosheets prepared from $\mathrm{P} \mathrm{BMA}_{227}-b-\mathrm{PS}_{187}$ block copolymer. The mixture was subsequently stirred at room temperature for two days, then the unreacted TFA was removed by centrifugation and washed with methanol. The white precipitate after centrifugation was re-dispersed into $\mathrm{KOH}$ aqueous solution $(1.0 \mathrm{M})$ and then dialyzed against neat water until the $\mathrm{pH}$ of water was ca. 7 . The final sample was characterized by TEM, SEM and FT-IR. 


\section{Results and discussion}

\subsection{Synthesis of PtBMA-b-PS block copolymers}

Six P $t$ BMA- $b$-PS block copolymers with the same P $t$ BMA block but varied PS were obtained by RAFT polymerization with $\mathrm{P} \mathrm{BMA}_{227}$ macroRAFT agent as shown in Scheme S1 (in Supporting Information). The block ratio and molecular weight of copolymers were calculated according to the ${ }^{1} \mathrm{H}$ NMR spectra in $\mathrm{CDCl}_{3}$. The typical ${ }^{1} \mathrm{H}$ NMR spectrum of PtBMA-b-PS block copolymer was shown in Fig. S1A (in Supporting Information). The polymeric degree of PS segment was calculated by comparing the integration ratio of the proton resonances derived from two segments like the peaks $\mathrm{g}, \mathrm{f}, \mathrm{e}(5 \mathrm{H}$, phenyl ring) from PS segment and the peak c $(9 \mathrm{H}$, tert-butyl group) from $\mathrm{P} t \mathrm{BMA}$ segment. Their respective SEC traces were recorded in Fig. S1B (in Supporting Information). The properties of block copolymers were collected in Table 1 . The polydispersities $\left(M_{\mathrm{w}} / M_{\mathrm{n}}\right)$ of all the copolymers were between 1.12 and 1.15 , indicating a well-controlled polymerization.

Table 1. Characteristics of PtBMA-b-PS block copolymers and the morphology of their microphase-separated samples.

\begin{tabular}{lcccc}
\hline Block copolymer & $\begin{array}{c}M_{\mathrm{n}}(\mathrm{NMR}) \\
\mathrm{kg} \mathrm{mol}^{-1} a\end{array}$ & $M_{\mathrm{w}} / M_{\mathrm{n}}{ }^{b}$ & $f_{\mathrm{PS} \mathrm{wt}} \%^{a}$ & Morphology $^{c}$ \\
\hline $\mathrm{P} \mathrm{BMA}_{227}-b-\mathrm{PS}_{160}$ & 48.9 & 1.15 & 34.0 & $\mathrm{C}$ \\
$\mathrm{P} \mathrm{BMA}_{227}-b-\mathrm{PS}_{180}$ & 50.9 & 1.14 & 36.7 & $\mathrm{C}$ \\
$\mathrm{P}_{\mathrm{B} \mathrm{BMA}_{227}-b-\mathrm{PS}_{187}}$ & 51.7 & 1.12 & 37.6 & $\mathrm{PL}$ \\
$\mathrm{P}_{\mathrm{B} \mathrm{BMA}_{227}-b-\mathrm{PS}_{200}}$ & 53.0 & 1.13 & 39.2 & $\mathrm{PL}+\mathrm{L}$ \\
$\mathrm{P}_{\mathrm{B} \mathrm{BMA}_{227}-b-\mathrm{PS}_{234}}$ & 56.6 & 1.15 & 43.0 & $\mathrm{~L}$ \\
$\mathrm{P}_{\mathrm{B} \mathrm{BMA}_{227}-b-\mathrm{PS}_{320}}$ & 65.5 & 1.13 & 50.8 & $\mathrm{~L}$ \\
\hline
\end{tabular}

${ }^{a}$ Estimated from monomer conversion by ${ }^{1} \mathrm{H}$ NMR spectra. ${ }^{b}$ The polydispersity $\left(M_{\mathrm{w}} / M_{\mathrm{n}}\right)$ of the block copolymers were determined by SEC with PS standard in DMF eluent. ${ }^{c}$ Evaluated by TEM images (in Fig. 4) of the dispersions of block copolymer microphase-separated samples in methanol, respectively. $\mathrm{L}=$ Lamella, $\mathrm{PL}=$ Perforated Lamella, $\mathrm{C}=$ Cylinder. 


\subsection{Formation of $P L$ microphase structure from $P t B M A_{227}-b-P S_{187}$ copolymer}

For the $\mathrm{P} t \mathrm{BMA}_{227}-b-\mathrm{PS}_{187}$ block copolymer $\left(M_{\mathrm{w}} / M_{\mathrm{n}}=1.12\right.$ and $\left.f_{\mathrm{PS}}=37.6 \mathrm{wt} \%\right)$, a THF solution of the block copolymer was cast onto a Teflon substrate, and the solvent was evaporated slowly at room temperature to yield a polymer film with a thickness of ca. $0.5 \mathrm{~mm}$, which was then thermally annealed at $155^{\circ} \mathrm{C}$ (above $T_{\mathrm{g}}$ of $\mathrm{P} t \mathrm{BMA} \sim 115^{\circ} \mathrm{C}$ and PS $\sim 100{ }^{\circ} \mathrm{C}$ ). The structure of the bulk microphase-separated sample was investigated by SAXS and TEM, respectively. As shown in Fig. 1A, a scattering pattern of $q^{*}: 2 q^{*}: 3 q^{*}: 4 q^{*}: 6 q^{*}$ was observed. It seems that the overall lamellar structure had been obtained. The average domain spacing value $d$ was calculated to be $29.4 \mathrm{~nm}\left(d=2 \pi / q^{*}\right)$ from the primary peak position value at the lowest $q^{*}$. Also, an extended tail seems to be exhibited in the right side of primary peak signal (the gray region in Fig. 1A). According to the literature [16], the perforated lamellar (PL) phase may give a weak shoulder signal (at $q=1.08 q^{*}$ ) near the main peak position. However, we could not clearly observe it although the sample was subjected to analysis for several times. It should be attributed to the non-uniform perforation which is typically associated with polydispersity of block copolymer and relatively poor long range order in the microphase-separated film with a larger thickness $[8,22]$. Further, a microtomed slice of the microphase-separated sample was observed by TEM. As seen in Fig. 1B (no staining) and Fig. 1C (PS component was selectively stained with $\mathrm{RuO}_{4}$ vapor prior to TEM observation.), an alternating lamellar structure with a periodic width of ca. $29.8 \mathrm{~nm}$ was observed. The light layers are attributed to the P $t$ BMA phases and the dark layers are the PS phases due to its higher electron

contrast. It is noteworthy that the PS layers seem not homogenous and, actually, appear with a bamboo-like pattern. It implies that there are PtBMA domains dispersed in PS layers [22]. 

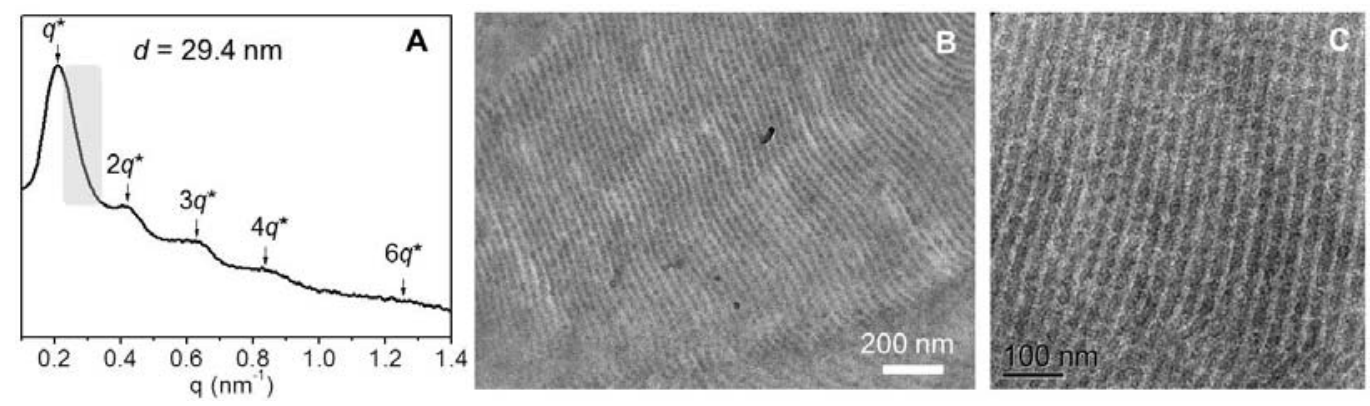

Fig. 1. (A) SAXS curve and (B, C) TEM image of a microtomed slice of the $\mathrm{P} t \mathrm{BMA}_{227}-b$ - $\mathrm{PS}_{187}$ block copolymer bulk: (B) no staining, (C) staining with $\mathrm{RuO}_{4}$ for $40 \mathrm{~min}$.

\subsection{Dispersion of microphase-separated samples}

In order to further confirm the PL phase structure, the annealed film sample of $\mathrm{P} t \mathrm{BMA}_{227}-b-\mathrm{PS}_{187}$ copolymer bulk was dispersed in methanol to give sheetlike particles for observation. Previously, we confirmed that the lamellar structure of PS segment-bearing block copolymers could be dispersed in methanol to give isolated sheetlike particles with hydrophobic PS layers sandwiched by the methanol-soluble segments at room temperature [26-29]. Namely, the layer structure of the dispersed particles is inherited from their bulk structure. The glassy PS layers are strong enough to keep their non-spherical shape in the poor solvent of PS. This property is very useful not only to confirm its bulk structure but also to generate unique individual block copolymer nanoobjects that cannot be obtained through a solution self-assembly approach. After stirring for one day, the bulk material disappeared completely; meanwhile, a white opaque solution was obtained, suggesting formation of the sheetlike morphology [26, 29]. The dispersion was dropped onto a carbon coated copper grid and, after staining with $\mathrm{RuO}_{4}$, it was subjected for TEM observation. As shown in Fig. 2A and 2B, the morphology shows an irregular sheetlike shape. It is noteworthy that the gray surface of each sheet has a pattern of minute white dots. Since the PS domains were selectively stained, the dark regions were PS phases and white dots were PtBMA. Thus it is proved that the PS layers have dispersed PtBMA domains. The average periodic distance and the diameter of the white dots, PtBMA, 
can be measured to be ca. 31 and $12 \mathrm{~nm}$. The inset pattern in Fig. 2B was the Fourier-transformed image and a symmetric hexagonal pattern was given. Therefore, the $\mathrm{P} t \mathrm{BMA}_{227}-b-\mathrm{PS}_{187}$ bulk sample with a hexagonal PL structure was confirmed.
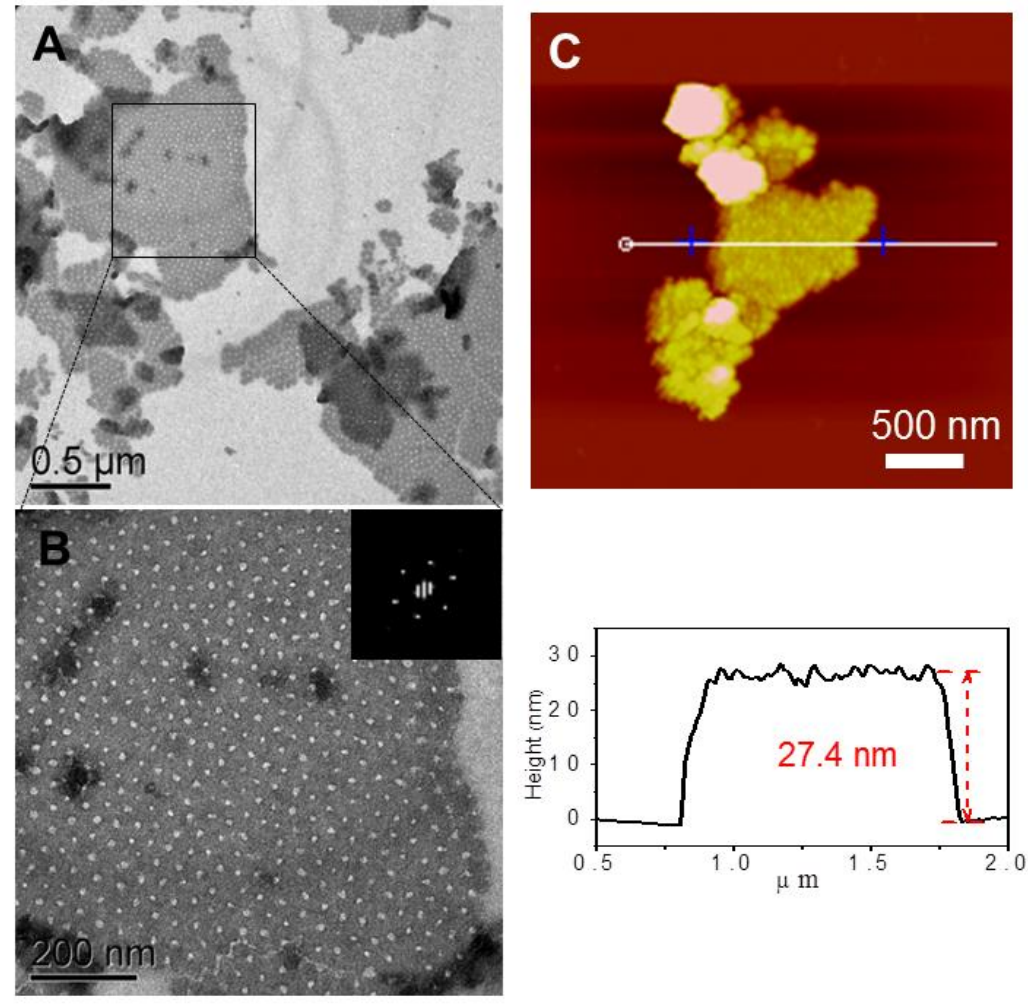

Fig. 2. (A, B) TEM images of isolated perforated nanosheets from microphase-separated $\mathrm{P} t \mathrm{BMA}_{227}-b-\mathrm{PS}_{187}$ sample stained with $\mathrm{RuO}_{4}$ vapor for 40 min. Inset in B was Fourier-transferred image from pattern of nanosheet. (C) AFM height image of perforated nanosheets (top) and height profile along the white line in AFM image (bottom).

The sheets dispersed in methanol were also characterized by atomic force microscopy (AFM). From Fig. 2C, it is noticed that the surface of sheets is not smooth and the height image shows a zig-zag pattern. This phenomenon may be understood from the structure of PL phase. Due to the packing character, the height of PtBMA passages is relatively lower than that of the PtBMA on both sides of PS phase. Unfortunately, owing to the resolution of AFM tip and the softness of PtBMA, it is hard to observe the pattern directly by AFM. The averaged height of sheets is ca. 27.4 
$\mathrm{nm}$, which is nearly one periodic length from SAXS, indicating a good dispersion of nanosheets. Furthermore, the dispersed nanosheets were studied by SEM observation and many pores were observed along the sheets (Fig. 3). From the area highlighted by a black circle, ordered passages are found. However, it should be noted that some nanopores became unclear and formed vague lines on the surface, it seems that the $\mathrm{P} t \mathrm{BMA}$ polymer chains tethered on surface of the nanosheets concealed part of pores under dry state. As indicated by white circles, there are also some areas with larger and even broken pores. It should be produced during sample preparation for SEM observation.

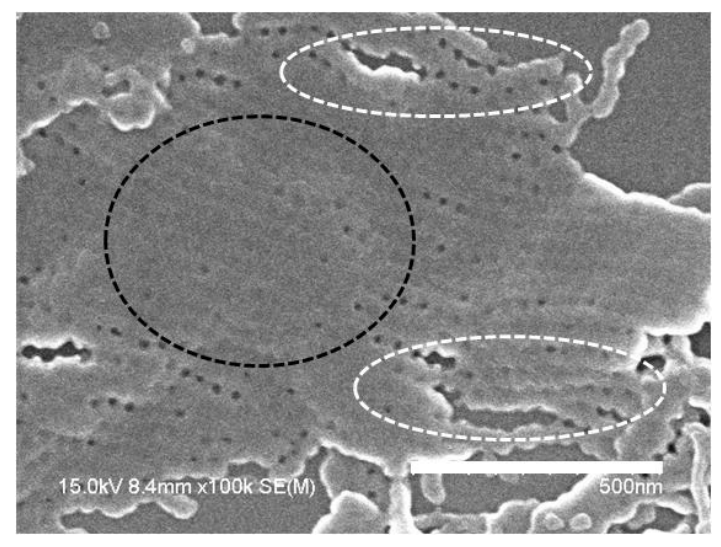

Fig. 3. SEM image of the isolated perforated nanosheet. The black circle shows the ordered passages along the sheets, and the white circles display the larger and broken porous structure.

\subsection{The effect of copolymer composition on microphase-separated structure}

It is curious to explore how the segment fraction of the copolymers influences the microphase-separation development. We also synthesized other five PtBMA- $b$-PS block copolymers using the same macroRAFT agent $\mathrm{P} \mathrm{BMA}_{227}$. Thus six samples with the same $\mathrm{P} t \mathrm{BMA}$ chain length but varied PS length, $\mathrm{P} t \mathrm{BMA}_{227}-b-\mathrm{PS}_{\mathrm{n}}(\mathrm{n}=160$, 180, 187, 200, 234, and 320), were obtained (Table 1). These block copolymers were microphase-separated according to the same procedure of $\mathrm{P} \mathrm{BMA}_{227}-b-\mathrm{PS}_{187}$ and their morphologies were confirmed by dispersing method. The TEM images of their

dispersions were shown in Fig. 4, respectively. For the samples of $\mathrm{P} t \mathrm{BMA}_{227}-b-\mathrm{PS}_{160}$ 
$\left(f_{\mathrm{PS}}=34.0 \mathrm{wt} \%\right)$ and $\mathrm{P} t \mathrm{BMA}_{227}-b-\mathrm{PS}_{180}\left(f_{\mathrm{PS}}=36.7 \mathrm{wt} \%\right)$, as seen in Fig. $4 \mathrm{~A}$ and $4 \mathrm{~B}$, they all formed cylinder-shaped dispersions, demonstrating that their bulk materials have a $\mathrm{C}$ phase. The nanofibers with ca. $30.5 \mathrm{~nm}$ in diameter and several micrometers in length were obtained. With a slight increase of PS chain length, the sample of $\mathrm{P} t \mathrm{BMA}_{227}-b-\mathrm{PS}_{187}\left(f_{\mathrm{PS}}=37.6 \mathrm{wt} \%\right)$ forms the ordered PL phase (Fig. 4C). When the PS fraction further increased to $39.2 \mathrm{wt} \%$, i.e., $\mathrm{P} t \mathrm{BMA}_{227}-b-\mathrm{PS}_{200}$, the similar perforated sheets were observed as shown in Fig. 4D. However, it is noteworthy that some areas without pattern were also found (highlighted by black circles). It implies the coexistence of PL and L phases, the transformation region from PL phase to L phase with the increase of PS fraction. As further increase of PS fraction to $43.0 \mathrm{wt} \%$ (Fig. 4E), $\mathrm{P} t \mathrm{BMA}_{227}-b-\mathrm{PS}_{234}$, and to $50.8 \mathrm{wt} \%$ (Fig. 4F), $\mathrm{P} t \mathrm{BMA}_{227}-b-\mathrm{PS}_{320}$, the sheetlike particles with the size of several micrometers but without patterned surface were obtained, indicating the formation of normal L phase in the bulks [26]. It is concluded that the PL phase is between $\mathrm{L}$ and $\mathrm{C}$ phase during bulk microphase separation. This result is consistent with the previous report on phase transition theory [23].
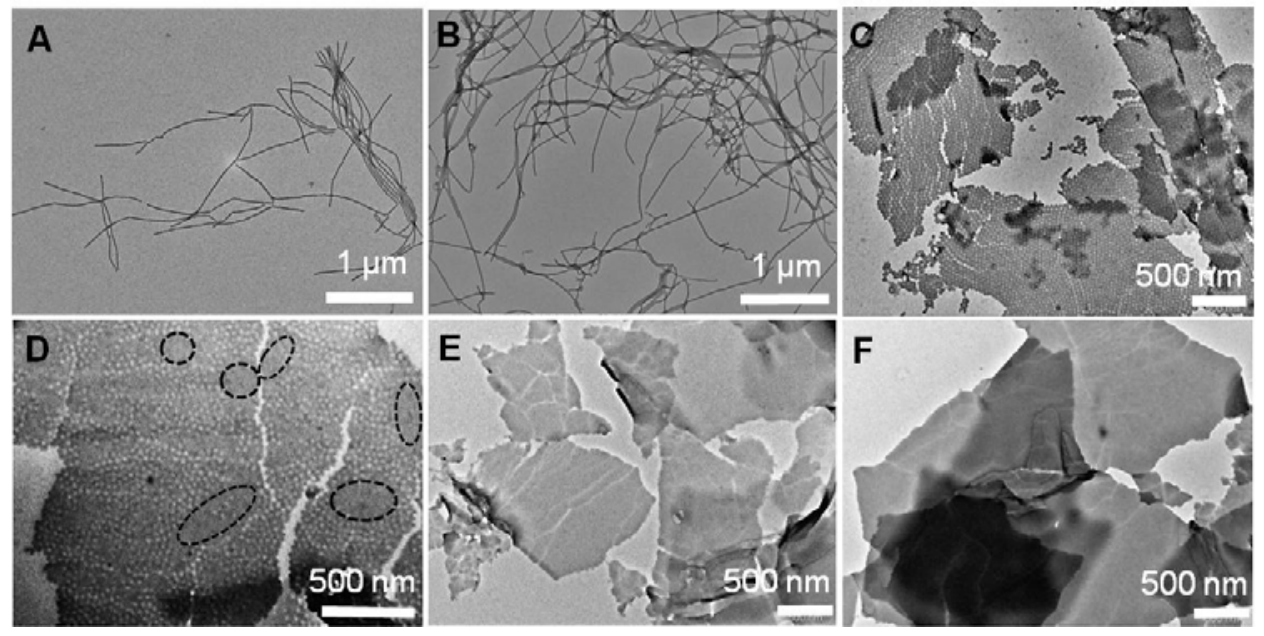

Fig. 4. TEM images of shaped nanoobjects dispersed in methanol from microphase-separated samples of six $\mathrm{P} \mathrm{BMA}_{227}-b$ - $\mathrm{PS}_{\mathrm{n}}$ block copolymers with varied mass fraction of PS: (A) $34.0 \mathrm{wt} \%$, (B) $36.7 \mathrm{wt} \%$, (C) $37.6 \mathrm{wt} \%$, (D) $39.2 \mathrm{wt} \%$, (E) $43.0 \mathrm{wt} \%$, and (F) $50.8 \mathrm{wt} \%$. The black circles in D show the non-patterned areas.

Above results indicate that the PL phase is generated from PtBMA- $b$-PS 
copolymers. The structure has an alternating structure of PtBMA and PS layers, among which the PS layer has a hexagonally arranged PtBMA perforation. Thus the PS sheet has a sieve sheetlike structure and the alternating P $t$ BMA layers are connected through ordered passages of PtBMA. Scheme 2 shows the cartooned sheet with a core/shell structure, in which the perforated PS core sheet is sandwiched by PtBMA layers.

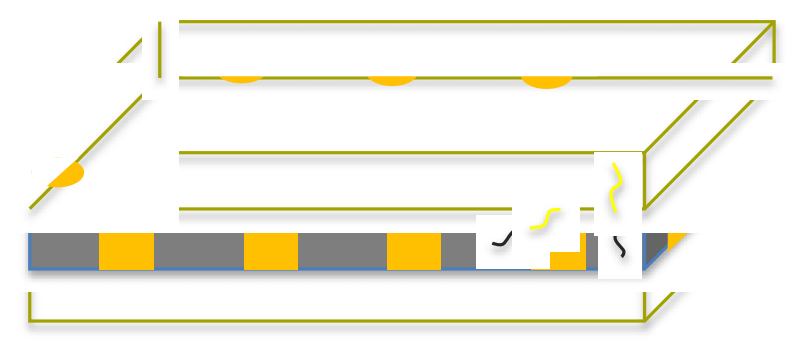

Scheme 2. Representation of core/shell perforated nanosheets of diblock copolymers. The yellow and deep gray colors represent the PtBMA and PS components.

\subsection{Water-dispersed perforated nanosheet}

Due to the robust glassy PS core, the sheet without chemical cross-linking is stable in methanol. Then we wonder if the PL sheets are strong enough for chemical transformation. A dispersion of PL sheets of $\mathrm{P} \mathrm{BMA}_{227}-b-\mathrm{PS}_{187}$ in methanol was treated with TFA in order to change the PtBMA to the PMAA at room temperature. The obtained white precipitate was then dispersed into an alkaline aqueous solution followed by dialysis against water. Almost complete hydrolysis was confirmed by FT-IR analysis given in Fig. S2 (in Supporting Information). The water-dispersed sample was characterized by TEM and SEM. As shown in Fig. 5A and 5B, it was found that the shape of whole nanosheet is kept intact and also the patterned structure is still remained. The TEM sample was not stained and thus the darker dot pattern is attributed to the presence of metal ions (PMAA-K ${ }^{+}$). Fig. 5C gives SEM image of the hydrolyzed PL sheets; it is hardly to find the pore-like structure, which should be caused by coverage of the PMAA- $\mathrm{K}^{+}$shell. This also provides a convenient route for further functionalization and modification of PL materials. 

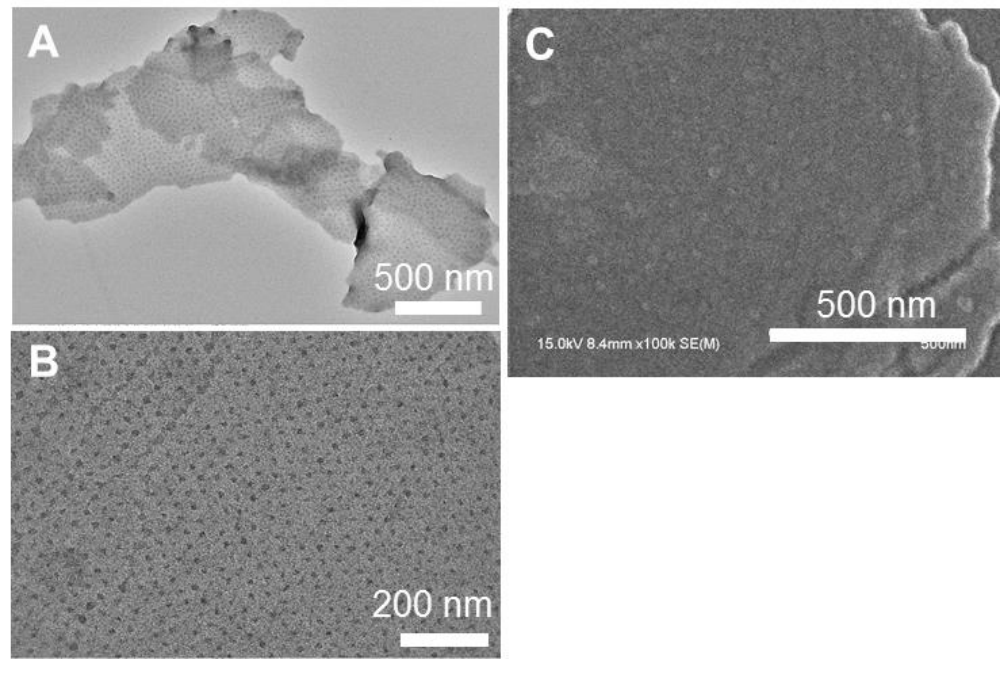

Fig. 5. (A, B) TEM images and (C) SEM image of hydrolyzed perforated nanosheets dispersed in water.

\section{Conclusion}

In summary, film materials having a perforated lamellar structure and isolated perforated polymer nanosheets with the core/shell structure were simply prepared from P $t$ BMA- $b$-PS diblock copolymer $\left(f_{\mathrm{PS}}=37.6 \mathrm{wt} \%\right)$. We believe that the PL bulks may be used to prepare unique porous materials, and the dispersed sheets with passages may be utilized as well-controlled ultrathin films for ion transportation, etc.

\section{Supplementary Data}

Synthetic route of PtBMA- $b$-PS block copolymer (Scheme S1), the typical ${ }^{1} \mathrm{H}$ NMR spectrum and SEC profiles of block copolymers (Fig. S1), and FT-IR analysis of perforated nanosheets and hydrolyzed perforated nanosheets (Fig. S2). These supplementary data are available on the website.

\section{Acknowledgements}

Financial support by the National Science Foundation of China (51533009), Guangdong Innovation Team Project (2013S086), and Guangdong Natural Science Foundation (2014A030312018) is greatly acknowledged. 


\section{References}

[1] Y.Y. Mai, A. Eisenberg, Self-assembly of block copolymers, Chem. Soc. Rev. 41 (2012) 5969-5985.

[2] S. Förster, T. Plantenberg, From self-organizing polymers to nanohybrid and biomaterials, Angew. Chem. Int. Ed. 41 (2002) 688-714.

[3] B. Nandan, A. Horechyy, Hairy core-shell polymer nano-objects from self-assembled block copolymer structures, ACS Appl. Mater. Interfaces 7 (2015) 12539-12558.

[4] F.H. Schacher, P.A. Rupar, I. Manners, Functional block copolymers: nanostructured materials with emerging applications, Angew. Chem. Int. Ed. 51 (2012) 7898-7921.

[5] S. Ludwigs, A. Böker, A. Voronov, N. Rehse, R. Magerle, G. Krausch, Self-assembly of functional nanostructures from ABC triblock copolymers, Nat. Mater. 2 (2003) 744-747.

[6] T. Ito, Block copolymer-derived monolithic polymer films and membranes comprising self-organized cylindrical nanopores for chemical sensing and separations, Chem. Asian J. 9 (2014) 2708-2718.

[7] J. Martín, M. Martín-González, A. Del Campo, J.J. Reinosa, J.F. Fernández, Ordered arrays of polymeric nanopores by using inverse nanostructured PTFE surfaces, Nanotechnology 23 (2012) 385305.

[8] N.A. Lynd, A.J. Meuler, M.A. Hillmyer, Polydispersity and block copolymer self-assembly, Prog. Polym. Sci. 33 (2008) 875-893.

[9] T.A. Shefelbine, M.E. Vigild, M.W. Matsen, D.A. Hajduk, M.A. Hillmyer, E.L. Cussler, F.S. Bates, Core-shell gyroid morphology in a poly(isoprene-blockstyrene-block-dimethylsiloxane) triblock copolymer, J. Am. Chem. Soc. 121 (1999) 8457-8465.

[10] Y.D. Xia, J.Z. Chen, T.F. Shi, L.J. An, Self-assembly of linear rod-coil multiblock copolymers, Chin. J. Polym. Sci. 31 (2013) 1242-1249.

[11]F.H. Schacher, H. Sugimori, S. Hong, H. Jinnai, A.H.E. Müller, Tetragonally perforated lamellae of polybutadiene-block-poly(2-vinylpyridine)-blockpoly(tert-butyl methacrylate) (BVT) triblock terpolymers in the bulk: preparation, cross-linking, and dissolution, Macromolecules 45 (2012) 7956-7963. 
[12]E. Betthausen, M. Dulle, C. Hanske, M. Mu ller, A. Fery, S. Fo rster, F.H. Schacher, A.H.E. Mu ller, Nanoporous sheets and cylinders via bulk templating of triblock terpolymer/homopolymer blends, Macromolecules 47 (2014) 6289-6301.

[13]I.W. Hamley, K.A. Koppi, J.H. Rosedale, F.S. Bates, K. Almdal, K. Mortensen, Hexagonal mesophases between lamellae and cylinders in a diblock copolymer melt, Macromolecules 26 (1993) 5959-5970.

[14]D.A. Hajduk, R.M. Ho, M.A. Hillmyer, F.S. Bates, K. Almdal, Transition mechanisms for complex ordered phases in block copolymer melts, J. Phys. Chem. B 102 (1998) 1356-1363.

[15]I.W. Hamley, V. Castelletto, O.O. Mykhaylyk, Z. Yang, Mechanism of the transition between lamellar and gyroid phases formed by a diblock copolymer in aqueous solution, Langmuir 20 (2004) 10785-10790.

[16]I. Vukovic, G. Brinke, K. Loos, Hexagonally perforated layer morphology in PS- $b$-P4VP(PDP) supramolecules, Macromolecules 45 (2012) 9409-9418.

[17]H.W. Park, K. Im, B. Chung, M. Ree, T. Chang, K. Sawa, H. Jinnai, Direct observation of HPL and DG structure in PS- $b$-PI thin film by transmission electron microscopy, Macromolecules 40 (2007) 2603-2605.

[18]D.M. Lambreva, R. Opitz, G. Reiter, P.M. Frederik, W.H. de Jeu, Morphology of an asymmetric ethyleneoxide-butadiene diblock copolymer in bulk and thin films, Polymer 46 (2005) 4868-4875.

[19]C.S. Thomas, M.J. Glassman, B.D. Olsen, Solid-state nanostructured materials from self-assembly of a globular protein-polymer diblock copolymer, ACS Nano 5 (2011) 5697-5707.

[20]H.G. Schoberth, V. Olszowka, K. Schmidt, A. Böker, Effect of electric fields on block copolymer nanostructure, Adv. Polym. Sci. 227 (2010) 1-31.

[21]J. Listak, W. Jakubowski, L. Mueller, A. Plichta, K. Matyjaszewski, M.R. Bockstaller, Effect of symmetry of molecular weight distribution in block copolymers on formation of "Metastable" morphologies, Macromolecules 41 (2008) 5919-5927.

[22]K.K. Tenneti, X.F. Chen, C.Y. Li, Y.F. Tu, X.H. Wan, Q.F. Zhou, I. Sics, B.S. 
Hsiao, Perforated layer structures in liquid crystalline rod-coil block copolymers, J. Am. Chem. Soc. 127 (2005) 15481-15490.

[23]S. Förster, A.K. Khandpur, J. Zhao, F.S. Bates, I.W. Hamley, A.J. Ryan, W. Bras, Complex phase behavior of polyisoprene-polystyrene diblock copolymers near the order-disorder transition, Macromolecules 27 (1994) 6922-6935.

[24]A.K. Khandpur, S. Farster, F.S. Bates, I.W. Hamley, A.J. Ryan, W. Brass, K. Almdal, K. Mortensen, Polyisoprene-Polystyrene diblock copolymer phase diagram near the order-disorder transition, Macromolecules 28 (1995) 8796-8806.

[25]G. Moad, E. Rizzardo, S.H. Thang, Radical addition-fragmentation chemistry in polymer synthesis, Polymer 49 (2008) 1079-1131.

[26]J.L. Qin, Y.M. Chen, D.D. Yan, F. Xi, Dispersible shaped nanoobjects from bulk microphase separation of high $T_{\mathrm{g}}$ block copolymers without chemical cross-linking, Macromolecules 43 (2010) 10652-10658.

[27]Y.M. Chen, Shaped hairy polymer nanoobjects, Macromolecules 45 (2012) 2619-2631.

[28]J.L. Qin, Y.M. Chen, Shaped core-shell nanoparticles prepared from self-assembly of block copolymers, Acta Polym. Sin. 6 (2011) 572-585.

[29]D.D. Yao, Y.M. Chen, R.H. Jin, Different dimensional silica materials prepared using shaped block copolymer nanoobjects as catalytic templates, J. Mater. Chem. B 3 (2015) 5786-5794. 


\section{Graphical Abstract}

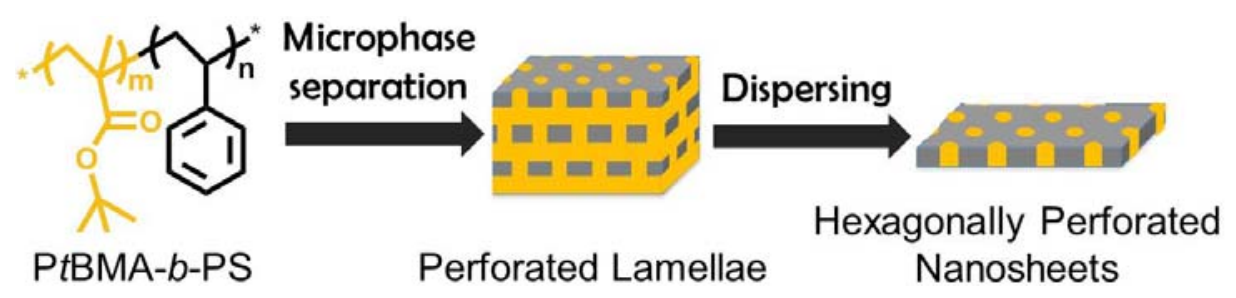

